\title{
A Review on Lifetime Enhancement in Wireless Sensor Networks
}

\author{
Aparna Gopinath P.K, Grace John \\ PG Scholar,Dept of ECE VJEC,Chemperi \\ Assistant Professor,Dept of ECE VJEC,Chemperi
}

\begin{abstract}
Wireless sensor networks are used to monitor physical or environmental conditions like temperature, pressure, sound etc.Increasing the lifetime of wireless sensor networks is a major challenge because the nodes are equipped with low power battery.Energy efficient routing helps in increasing the lifetime of sensor nodes by maximizing the overall performance of the nodes. Generally in routing algorithm best path is chosen for transmission from source to destination,but if the same path is used for a long period of time for quick transmission time the nodes in the selected path will get drained fast.This paper discusses about the different methods used for increasing the lifetime of wireless sensor networks.

Index terms: sensor node, wireless sensor networks, routing.
\end{abstract}

\section{Introduction}

Wireless sensor networks are collections of compact size and relatively inexpensive computational nodes that measure local environmental conditions or other parameters and forward such information to the base station for appropriate processing. The basic unit in a sensor network is a sensor node. Wireless sensor networks can sense the environment, communicate with neighbouring nodes and can also perform basic computations on the data being collected.Recent developments in sensor technology and wireless communication have helped in the deployment of large scale wireless sensor networks for a variety of applications including environmental monitoring of habitat,data collection of temperature, pressure, sound ,humidity, light,vibration etc.For such type of applications hundreds or thousands of low cost sensor nodes can be deployed over the area to be monitored.In data gathering sensor network each sensor node must periodically report its sensed data to the sink.

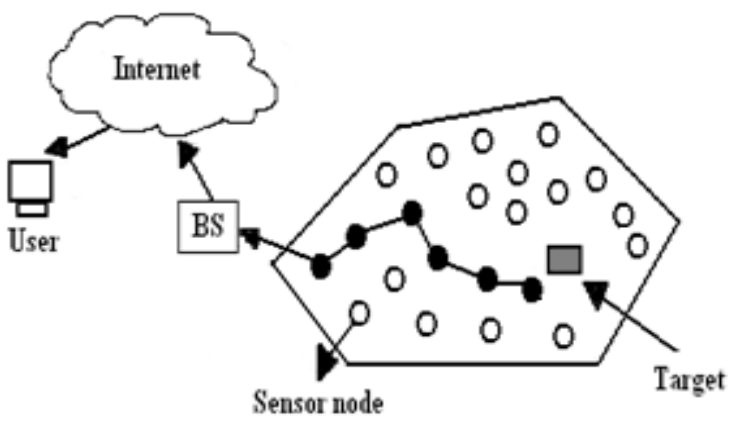

Fig.1.Sensor network architecture

The sensor nodes are generally powered by small inexpensive batteries. Therefore energy consumption should be managed in an efficient way to maximize the post deployment network lifetime.If there is long distance between the sensor and sink ,transmission is not energy efficient since the transmission power is proportional to the square or quadruple of the transmission distance.Multihop routing is performed than sensor to sink direct transmission for long distance as more energy could be saved.But multihop routing cause overuse of the nodes close to the sink and make them run out of energy quickly. Therefore unbalanced energy consumption[3] is an important problem in direct transmission and multihop routing schemes.It can cause early collapse of the network due to the death of some critical nodes which results in significant reduction of network lifetime.Each node sends its data to the sink without any relay in direct transmission mode and this mode helps in avoiding the relay burden for the nodes close to the sink.In hop by hop transmission mode each node forwards the data to its next level hop neighbours and this helps in reducing the burden of long distance transmission for nodes far away from the sink. Therefore even energy consumption among all nodes can be obtained by properly allocating the amount of data transmitted in the two modes. An energy cost function has been used in [6] which considers initial energy,residual energy and the required transmission energy along the route.In modified bellman ford algorithm [8] route with minimum cost is selected from all available routes in an iterative manner.Different battery levels in a clustering routing is assigned to the nodes in[10]. There is an improvement in the performance 
compared to the uniform level battery distribution. This paper discusses about the different methods used for improving the network lifetime.Section II discusses about energy aware sink relocation method.Section III describes about balancing energy consumption method and section IV about a-star algorithm and fuzzy approach.Paper is concluded in section V.

\section{Energy aware sink relocation method}

In a wireless sensor network sensor nodes has limited energy .So to increase the lifetime of wireless sensor network energy of sensor nodes has to be conserved.Sensor nodes after sensimg the data will deliver it to the sink through multihopping.Nodes near the sink will consume more battery power than others.Due to this these nodes will fastly drain out their battery energy and reduce the lifetime of the network.Sink relocation is an efficient method for enhancing the lifetime of the network.Energy aware sink relocation method is used here.A relocatable sink helps in prolonging the lifetime of the network by avoiding remaining at a certain location for a long time which may reduce the lifetime of nearby sensor nodes.The sink relocation method has two parts. The first part is to determine whether to trigger the sink relocation by determining whether a relocation condition is met or not.The second part determines which direction sink is heading in and the relocation distance.For relocation condition sink periodically collect the residual battery energy of each sensor node in the wireless sensor network.Then maximum capacity path routing protocol is used to find the maximum capacity path with respect to each sensor neighbor of the sink.For each maximum capacity path maximum capacity value is found.Sink relocation occurs when the maximum capacity value drops below a threshold value.

The sink relocation mechanism takes into account the residual battery energy of the sensor node and then drive the sink to a position with a large amout of residual energy compared to others.The maximum capacity routing algorithm has three steps.Layering the graph into a layered network,determining the maximum capacity path for each node,routing is performed and updation of residual energy.In the energy aware sink relocation method the transmission range of each sensor node is tuned according to the residual battery energy.Energy aware sink relocation has two parts energy aware transmission range adjusting and sink relocation.If a sensor node has large transmission range then its number of neighbors will be more, its drawback is long distance transmission will consume more battery energy of the sensor node.If the range of the node is less it doesnot help too much routing and it can conserve the residual battery energy.A node with more residual battery energy can use a large transmission range to reduce the routing path.On the other hand a node with less residual battery energy can tune its transmission range to be small to conserve its battery energy.Thus adaptable transmission mechanism can enhance the lifetime of a sensor node and hence the lifetime of the network.Sink relocation occurs when the residual battery energy of the nearby sensor node of the sink becomes small and sink will relocate to a new position which can increase the network lifetime.

\section{Balanced energy consumption method}

Unbalanced energy consumption is an inherent problem in wireless sensor network which can reduce the network lifetime.Corona based network division and mixed routing strategy are combined with data aggregation.In data gathering sensor network each sensor node periodically senses the data and sends it to the sink.Sensor nodes are powered by small inexpensive battery,therefore energy consumption should be managed efficiently to

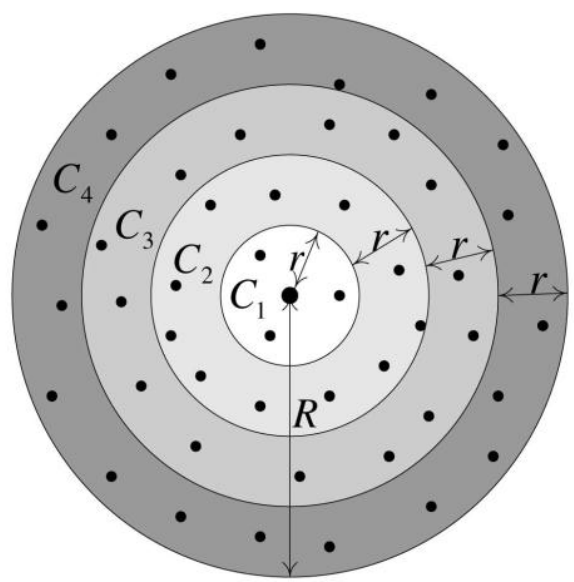

Fig.2.Illustration of network division

maximize network lifetime.Fully localizedzone based routing is used.Coronas are subdivided into zones.Optimal number of coronas needed to maximize the network lifetime is computed.The network is divided into coronas centered at the sink with equal width.All nodes use the same probability for direct transmission and 
hop by hop transmission.The energy consumption balancing is divided into intra corona energy consumption balancing and inter corona energy consumption balancing.In intra corona energy consumption balancing each corona is divided to evenly distribute the amount of data received by nodes in each corona.In inter corona energy consumption balancing amount of data for direct transmission and hop by hop transmission is divided optimally.Balanced energy consumption is obtained by optimally distributing the amount of data for hop by hop and direct transmission at each node.All nodes in the same corona use same transmission range for direct transmission and same transmission range for hop by hop transmission.In zone based routing each corona is divided into subcoronas and each subcorona is further divided into zones.Energy spent by transmission is proportional to the square of the transmission distance.For nodes close to the sink direct transmission is prefered.When energy consumption is balanced all nodes have same energy consumption.

\section{A-star algorithm and fuzzy approach}

This method determines an optimal routing path from source to destination with highest remaining battery power,minimum number of hops and minimum traffic loads.A combination of fuzzy approach and a-star algorithm is used.Generally in routing algorithm best path is chosen for transmission from source to destination.but if the same path is used for a long period of time for quick transmission time the nodes in the selected path will get drained fast.The optimal routing path is calculated by the base station and broadcasts it the nodes in the network. All the sensor nodes are distributed randomly and they have the same maximum transmission range.

Fuzzy approach

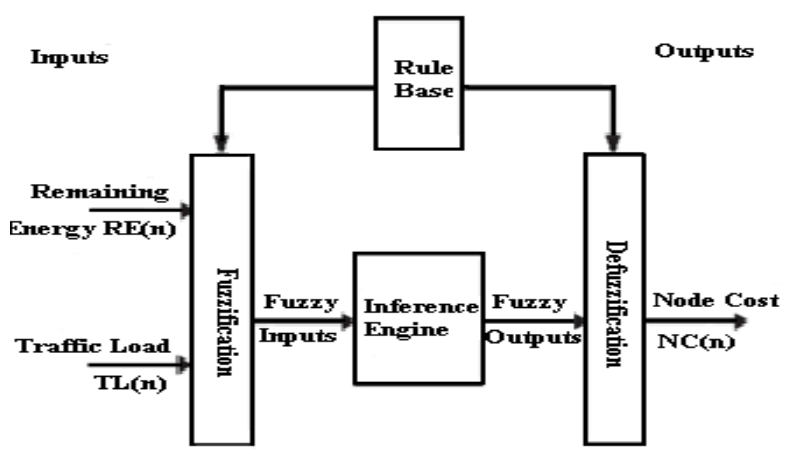

Fig.3.Fuzzy structure with two inputs and one output

The dynamic behaviour of a system is defined by a set of fuzzy rules based on the knowledge of a human expert.These rules are of the general form if(antecedent) then(consequent).Anteced-

ents of a fuzzy rule use logic operations to form a combhination of fuzzy sets.The heart of a

fuzzy system are the rules.It is provided by human experts .It can be extracted from numerical data also.The rules can be expressed as a collection of if then statements.Antecedents of a fuzzy rule form the input fuzzy space and consequents form the output fuzzy space.A fuzzy system has four components namely fuzzification,rulebase,inferenceengine,defuzzification.In fuzzification crisp inputs are mapped to their fuzzy representation.In this membership functions like gaussian,triangular,trapezoidal are involved.In the inference engine the fuzzified inputs are mapped to the rule base to produce fuzzy output. In defuzzification the output of a fuzzy rule is mapped to crisp output.

\section{A-star algorithm}

A-star algorithm is an efficient algorithm for finding low cost path.It is one of the best search algorithm.It uses an evaluation function defined as $f(n)=g(n)+h(n)$

where $g(n)$ is actual cost from the start node to the node $n$ and $h(n)$ is the estimated cost of the optimal path from node $\mathrm{n}$ to the destination node.A-star algorithm has two lists namely open list and closed list.Open list is a priority queue and nodes are arranged based on the value of the evaluation function.Closed list contains the list of nodes that has already been examined.Initially the open list contains the start node after one iteration it takes top of the priority list and checks whether it is the destination node.If its not the destination node then it finds the evaluation function of all adjacent nodes and adds it to the open list.It finds the path with lowest possible cost.It creates a tree structure to search the optimal path from a node to the base station. The evaluation function is given as

$\mathrm{f}(\mathrm{n})=\mathrm{c}(\mathrm{n})+(1 / \mathrm{M}(\mathrm{n}))$

$\mathrm{c}(\mathrm{n})$ is the cost of the node $\mathrm{n}$,it is calculated using fuzzy approach.Fuzzy approach takes into account remaining energy and traffic load of node n.M(n) is the shortest distance from the node $n$ to the base station.In the fuzzy approach the fuzzified values are determined by the inference engine.The inference engine has rule base.Rule 
base is a series of if then rules.The fuzzy implication operator used is and.This method of maximizing network lifetime is highly efficient .

\section{Conclusion}

This paper discussed about the different routing techniques for enhancing the lifetime of the wireless sensor network.Unbalanced energy consumption is an important problem in the sensor networks which is characterized by multihop routing and many to one pattern of the traffic. In sink relocation method when the residual battery energy of the nearby sensor node of the sink becomes less than a threshold value sink will relocate to a new position which can increase the network lifetime.Fuzzy approach and a-star algorithm are combined for determining the optimal routing path from source to destination.Optimal routing path is found by considering the highest remaining power in battery with least number of hops and without overhead of traffic loads.This method outperforms the other two methods as in this case it finds the optimal path by taking into consideration remaining battery energy, traffic load of the sensor node and shortest distance from the source node to the sink node.

Reference

[1] C Hua and T.P Yum,"Optimal routing and data aggregation for maximizing the lifetime of wireless sensor network", IEEE ACM , vol 16 no.4,pp 892-903,Aug 2008.

[2] J.N Alkaraki,A.E Kamal,"Routing techniques in Wireless sensor networks a survey",IEEE wirelss Communication, vol.11,no.6.pp.6-28, Dec 2004

[3] H. RKarkvandi, E. Pecht, and O.Yadid,'Effective Lifetime aware routing in wireless sensor netwo-rks ”,IEEE Sensors journal,vol 11,no 12,pp. 3359-3367,Dec 2011.

[4] K.Akkaya and M.Younis,"A survey of routing protocols in wireless sensor networks",Ad Hoc networks, vol.3,no.3,pp 325349 , may 2005

[5] S.Y Chiang and J.L Wang," Routing analysis Fuzzy logic systems in wireless sensor networks" IEEE transactions, vol.11,no.2.pp 2-26,Oct 2011

[6] C.Wu,R.Yuan,H.Zhou,"A novel load balanced And lifetime maximization routing protocol in wireless sensor network", IEEE transactions, vol 3 pp. 305-314

[7] Dr Sami Halawani, Abdul Waheed Khan," Sensor enhancement techniques in wireless sensor networks" Journal of computing,vol.2, may 2011

[8] Jongseok Park and Sartaj Sahni,"An online Heuristic for maximum lifetime routing in wireless Sensor network",IEEETransactions , vol.55,no.8, Aug 2006

[9] Kobi Cohen and Amir Leshem,"A time varying Opportunistic approach to lifetime maximization Of wireless sensor networks", IEEE Transactions vol.58,no.10,October 2010

[10] Junlin li,Ghassan Alkegib,"Network lifetime Maximization for estimation in mulhop wire less sensor network."IEEE Transactions, vol. 57,no.7,july 2009

[11] Ritesh Madan,Shuguang Cui,Sanjay Lall,"Cross layer design for lifetime maximization in wireless sensor network" IEEE Transactions, vol.5, no.11 Nov 2006

[12] Imad S Alshawi,Lianshan Yan,Wei Pan.Bin Luo "Lifetime Enhancement in wireless sensor netwo Rk using fuzzy approach and astar algorithm", IEEE Sensors journal,vol.12,October 2012

[13] K. M. Rana and M. A. Zaveri, "ASEER: A routing method to extend life of two-tiered wireless sensor network," International Journal of Advanced Smart Sensor network system vol 11,no.2,pp 1-16,Oct.2011. 